Sportwiss 2014 $\cdot 44: 240-241$

DOI 10.1007/s12662-014-0338-8

Online publiziert: 17. Juli 2014

c) Springer-Verlag Berlin Heidelberg 2014
Ernst-Joachim Hossner - Christian Vater

Institut für Sportwissenschaft, Universität Bern, Bern, Schweiz

\title{
Wörterbuch Bewegungs- und Trainingswissenschaft
}

Schiffer, J., \& Mechling, H. (2013). Wörterbuch Bewegungs- und Trainingswissenschaft $(2 .$, verb. u. erw. Aufl.). Köln: Strauß, 432 S., ISBN 978-3-86884-149-7, 34,80 EUR.

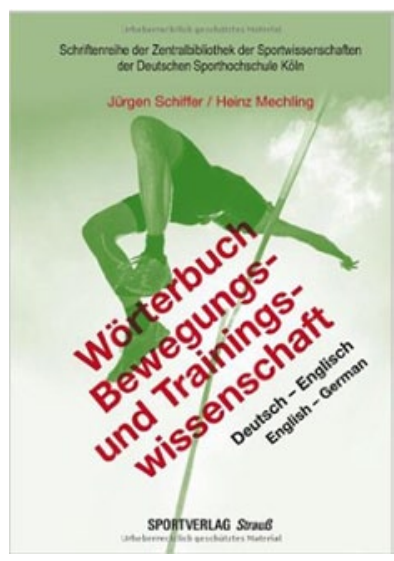

Nun - es mag dankbarere Aufgaben in der Wissenschaft geben, als ausgerechnet ein Wörterbuch zu besprechen. In einer akademischen Welt allerdings, in der zunehmend gefordert wird, auf Englisch zu publizieren, und in der es nur in den allerseltensten Fällen noch gelingen dürfte, ein Themenfeld hinreichend aufzubereiten, ohne dabei auf englischsprachige Literatur zurückzugreifen - in solch einer akademischen Welt sollte man es als Geschenk betrachten, wenn Fachkollegen sich die Mühe machen, Vorschläge für die deutsch-englische Übersetzung von Fachbegriffen anzubieten, die in keinem allgemeinen Wörterbuch aufzufinden sind. Denn, deutlich mehr noch als ein Wörterbuch zu besprechen: ein Wörterbuch zu verfassen, stellt definitiv keine sonderlich dankbare Aufgabe in der Wissenschaft dar...

Bei dem hier zu rezensierenden Buch handelt es sich um die 2013 von Jürgen Schiffer und Heinz Mechling vorgelegte
Neuauflage des Wörterbuchs Bewegungswissenschaft und Trainingswissenschaft, welches 2007, seinerzeit noch mit Christoph Igel als Drittautor, aus dem Saarbrücker eBuT-Projekt zum eLearning in der Bewegungs- und Trainingswissenschaft hervorgegangen war. Die in der Neuauflage vorgenommenen Erweiterungen zeigen sich darin, dass das Buch im Vergleich zur Erstauflage um etwa ein Drittel an Umfang zugenommen hat; die Autoren selbst berichten von einer „Erweiterung ... im deutschen Lemmabereich um 66 Prozent, im englischen Nebenlemmabereich um 100 Prozent" (S. 12). Mehr als quantitative Erweiterungen fallen jedoch die qualitativen Veränderungen ins Gewicht. Diese bestehen vor allem darin, dass i) die Wortliste um solche Begriffe bereinigt wurde, denen allein eine Bedeutung für die im eBuT-Projekt zusammengetragenen Texte zukam, dass ii) die Wortliste unter Hinzuziehung einschlägiger Standardtexte erheblich ausgeweitet wurde und dass iii) Umstrukturierungen für die Suche nach Mehrwort-Termini (wie bspw. „maximale aerobe Kapazität“) vorgenommen wurden. Im Resultat setzt sich das Wörterbuch zusammen aus einem Einleitungsteil samt Abkürzungsverzeichnis (26 Seiten), dem Kernteil des deutsch-englischen Wörterbuchs (222 Seiten), Verweisen für invertierte Formen (wie bspw. „Kapazität, maximale aerobe“, 72 Seiten), einem Index für die englischen Termini (wie bspw. „maximal aerobic capacity“, 104 Seiten) sowie einem Literaturverzeichnis und Autorenbiografien (8 Seiten). Beachtet man die Arbeit, die hinter jedem der auf all diesen Seiten aufgeführten Begriffe stecken dürfte, dann vermag das Vorgelegte in der $\mathrm{Zu}$ sammenschau schon sehr zu beeindrucken.
Zeigen wir uns für das hiermit gemachte Geschenk ehrlich dankbar, so wollen wir die uns angetragene Rezensionsaufgabe ernst nehmen. Anstelle des vage gehaltenen Lobs, dass ein Wörterbuch für den Umgang mit fremdsprachigen Texten hilfreich ist - wie könnte es anders sein - wollen wir daher etwas genauer hinschauen und stattdessen der ausdrücklichen Einladung der Autoren folgen, mit „kritische[n] Hinweise[n] ... [zu einer] Weiterentwicklung und Optimierung dieses Wörterbuchs bei[zu]tragen“ (S. 14). In diesem Sinne konnten wir drei kleinere Probleme ausmachen, die im Zuge einer etwaigen Neuauflage lösbar sein sollten, und ein größeres, welches sich aus grundlegenden Erwägungen heraus als unlösbar entpuppen dürfte. Beginnen wir mit den Detailanmerkungen:

a) Da deutschsprachigen Sportwissenschaftlerinnen und -wissenschaftlern beim Verfassen eines englischsprachigen Texts zugetraut werden sollte, dass sie über eine hinreichende Kenntnis der englischen Fachbegriffe verfügen, dürfte sich das Wörterbuch insbesondere für Sportstudierende bei der Bearbeitung englischsprachiger Texte als hilfreich herausstellen. Für diesen Zweck erweist sich das Buch jedoch nur bedingt als brauchbar, da in der aktuellen Fassung ein gesuchter englischer Begriff zuerst im englischen Index nachgeschlagen werden muss, um anschließend die dort angegeben Seite komplett im deutsch-englischen Wörterbuchteil zu durchsuchen. Für eine etwaige Neuauflage wird daher angeregt, statt eines bloßen Index einen vollständigen englisch-deutschen Wörterbuchteil aufzunehmen oder, falls dies vorgegebene Umfangsmaxima sprengen 
sollte, anstelle eines deutsch-englischen ein englisch-deutsches Wörterbuch vorzulegen.

b) Die von den Autoren gegebene Begründung für die Aufführung von Mehrwort-Termini in sämtlichen invertierten Formen ist zwar nachvollziehbar, schlägt sich mitunter aber in einer gewissen Unhandlichkeit nieder, wenn der Begriff zunächst im Verzeichnis der invertierten Formen nachgeschlagen werden muss, um dort den Verweis aufzufinden, an welcher Stelle im Wörterbuch der Begriff gesucht werden muss. Eingedenk der Tatsache, dass auch jede alternative Systematik Ansatzpunkte der Kritik bereitstellte, würden sich die Rezensenten aus diesem Grunde wünschen, dass in einer etwaigen Überarbeitung die Zahl der Querverweise drastisch reduziert und stattdessen einer einheitlichen Systematik gefolgt würde, etwa mit - in Wörterbüchern ja durchaus üblichen - Einordnung von substantivischen Mehrwort-Termini nach dem Substantiv (also bspw. von „maximale aerobe Kapazität“ einmalig unter „Kapazität, maximale aerobe").

c) Die Autoren beschreiben im Detail den Verifikationsprozess für die vorgeschlagenen Übersetzungen, der sich in einer ,akkuraten[n] englische[n] Übersetzung der Ausgangstermini [niederschlagen sollte], die von englischsprachigen Lesern weder künstlich noch als kulturell fremd empfunden werden dürfte“ (S. 10). Dies sollte den Autoren über den weitaus größten Teil des Buches hinweg gelungen sein; gleichwohl fallen einige Übersetzungsvorschläge auf, die dem jeweiligen Sprachgefühl entsprechen mögen, in fachlich geprägten Ohren aber nichtsdestoweniger etwas fremd klingen. So hätte man bspw. für die Übersetzung von „Bewegungskontrolle" zumindest auch mit der Option "motor control“ gerechnet, statt des Begriffspaars „WahrnehmungsMotorik-Kopplung“/,perceptual-motor coupling" eher (oder zumindest auch) das Paar „Wahrnehmungs-Handlungs-Kopplung “/,perception-action coupling" erwartet und sich als deutsche Entsprechung von ,augmented feedback" nicht unbedingt ,verstärkte Rückkopplung" gewünscht. Natürlich erschiene es kleinkrämerisch, solche Beispiele ins Zentrum dieser Besprechung zu rücken, denn bei über 8000 Einträgen würden sich wohl auch in der 100. Auflage des Buches noch Beispiele finden lassen, für die man die Güte der Übersetzung in Zweifel ziehen kann. Nichtsdestoweniger mag man hieraus die Konsequenz ziehen, bei einer etwaigen Neuauflage vor der Publikation eine größere Zahl von Fachvertreterinnen und -vertretern einzubinden, um die Übersetzungsvorschläge einer kritischen Prüfung $\mathrm{zu}$ unterziehen.

Die beiden erstgenannten Detailprobleme werden im Übrigen entschärft, wenn man (nach Freigabe durch Eingabe eines mitgelieferten Codes) anstelle des gedruckten Wörterbuchs dessen pdf-Version verwendet, denn dann erlaubt die elektronische Such-Funktion sowohl ein schnelleres Auffinden relevanter Stellen als auch die Verwendung des Wörterbuchs in beide Übersetzungsrichtungen. Da im Jahre 2014 der Online-Zugriff die typische Verwendung eines Wörterbuchs sein dürfte, könnte man bei einer etwaigen Neuauflage aber darüber nachdenken, zugunsten eines reduzierten Preises gänzlich auf eine gedruckte Fassung zu verzichten und zudem, statt der nur bedingt komfortablen pdf-Suche, ein interaktives Online-Tool anzubieten (etwa nach dem Vorbild von www.leo.org).

Bleibt - wie angekündigt - ein größeres Problem, eines, für das die Rezensenten keine Lösung anbieten können, nämlich das Problem, dass Begriffe ihre Bedeutung stets in einem theoretischen Kontext erhalten, Wörterbücher diese Begriffe aber - umständehalber - aus ihrem spezifischen Kontext herauslösen. Vor dem Hintergrund dieses Problems ist es sicherlich sprachlich korrekt, wenn man sowohl "recall schema“ als auch „recognition schema" mit „Erinnerungsschema" übersetzt; der Umstand, dass die Unterscheidung der beiden Schemata als (teil-)unabhängige Gedächtnisinstanzen ein ganz zentrales Merkmal der Sche- matheorie von Schmidt (1975) darstellt, gerät mit dieser gleichlautenden Übersetzung allerdings leicht aus dem Fokus. Und in ähnlicher Weise ist es sicherlich sprachlich korrekt, wenn man „kinästhetische Differenzierungsfähigkeit" mit „kinesthetic differentiation ability" übersetzt; nicht vergessen werden sollte dabei allerding, dass aufgrund der auf den deutschsprachigen Raum beschränkten Verwendung der Fähigkeitssystematik nach Hirtz (2007) nicht eingeweihte Leserinnen und Leser mit dem englischen Begriff nur wenig anfangen werden können. Wie gehabt: Lösbar scheint dieses Problem nicht zu sein; es verweist nur darauf, dass es für das Gelingen einer Übersetzung mehr bedarf als eines Wörterbuches - dass ein Wörterbuch erheblich zu diesem Gelingen beitragen mag, dürfte von dieser Feststellung unberührt bleiben.

\section{Korrespondenzadresse}

\section{Prof. Dr. E.-J. Hossner}

Institut für Sportwissenschaft

Universität Bern

Bremgartenstrasse 145

3012 Bern

ernst.hossner@ispw.unibe.ch

\section{Einhaltung ethischer Richtlinien}

Interessenkonflikt. Der korrespondierende Autor gibt an, dass kein Interessenkonflikt besteht.

\section{Literatur}

Hirtz, P. (2007). Koordinative Fähigkeiten und Beweglichkeit. In K. Meinel \& G. Schnabel (Red.: Schnabel, G., \& Krug, J.), Bewegungslehre - Sportmotorik. Abriss einer Theorie der sportlichen Motorik unter pädagogischem Aspekt (11. Aufl., S. 212-242). Aachen: Meyer \& Meyer.

Schmidt, R. A. (1975). A schema theory of discrete motor skill learning. Psychological Review, 82, 225260. 